\title{
Ictiofauna de embalses en cascada en el cauce de un río tropical andino
}

\section{Ichthyofauna of cascade reservoirs in the channel of a tropical Andean river}

\author{
Jonathan Álvarez-Bustamante ${ }^{1 *}$, Luz F. Jiménez-Segura ${ }^{1}$, Úrsula Jaramillo-Villa ${ }^{2}$
}

\begin{abstract}
Resumen
El Concepto de Discontinuidad Serial afirma que la formación de embalses en cadena dentro de los ríos genera cambios en la estructura de la biota acuática que se acumulan a lo largo del cauce. En el río Porce (Cuenca del río Magdalena-Cauca, Colombia) se formaron dos embalses en cadena, los embalses Porce II y Porce III. Para conocer la respuesta del ensamblaje de peces dentro del sector del río que fue embalsado se tomaron muestras de cuatro periodos de lluvias abundantes y cuatro de menores lluvias entre los años 2011 y 2013, en sitios dentro de los dos embalses seleccionados según su posición en el eje longitudinal (cola, transición y presa); para eliminar el efecto de la profundidad en cada sitio se tomaron muestras a dos profundidades (superficial-fondo). Se capturaron 15 especies; 12 en Porce II y 10 en Porce III. Se observaron diferencias espaciales en cuanto a la composición y estructura de especies de peces, tanto en el eje longitudinal (cola-presa), como en el vertical (superficie-fondo). En el embalse Porce II, la riqueza y la abundancia de individuos disminuyeron en dirección cola-presa y fue mayor en la superficie que en el fondo; en el embalse Porce III, aunque la riqueza presentó la misma tendencia en el eje longitudinal, la abundancia fue mayor en el fondo que en la superficie. Las características del ensamblaje no estuvieron asociadas a los periodos pluviométricos en ninguno de los dos embalses. Se concluye que la composición y estructura del ensamblaje está definida por las características morfológicas del embalse, así como la edad y tiempo de retención hidráulica.
\end{abstract}

Palabras claves: ensamblaje de peces, diversidad, peces de agua dulce, discontinuidad serial, río andino

\begin{abstract}
The Serial Discontinuity Concept affirms that the formation of reservoirs in cascade within rivers generates changes in the structure of the aquatic biota that accumulate along the basin. In the Porce River (MagdalenaCauca River Basin, Colombia) two reservoirs were formed in cascade, Porce II and Porce III. To know the response of the fish assemblage in the sector of the river that was dammed, we collected samples during four periods of abundant rains and four of lesser rains from 2011 to 2013 from sites inside both reservoirs selected according to position on the longitudinal axis (tail, transition and dam). To eliminate the effect of depth, at every site, we took samples from two depths, surface and bottom. 15 species were captured; 12 in Porce II and 10 in Porce III. Differences in the composition and structure of the species of fish were found to vary depending on the location along the river flow (from tail to dam), and also by the water depth (surface to river bottom). In Porce II reservoir, the richness and the abundance of fish diminished in direction tail to dam and the abundance decreased from surface to bottom. In Porce III though the species richness presented the same trend along the longitudinal axis, the abundance was greater on the bottom that at the surface. The characteristics of the assemblage were not associated with the rainfall periods in either reservoir. One concludes that the composition and structure of the assemblage are defined by the morphologic characteristics of the reservoir, as well as the age and time of hydraulic retention.
\end{abstract}

Keywords: fish assemblage, diversity, freshwater fishes, serial discontinuity, Andean river

1. Grupo de Ictiología, Instituto de Biología, Facultad de Ciencias Exactas y Naturales, Universidad de Antioquia, Medellín, Colombia.

2. Proyecto Delimitación de Ecosistemas Estratégicos: Paramos y Humedales. Instituto de Investigación en Biodiversidad Alexander von Humboldt, Bogotá, Colombia.

* Autor para correspondencia: <jonalvarez04@gmail.com>

Recibido: junio de 2017; aceptado: abril de 2018. 


\section{INTRODUCCIÓN}

Los embalses rompen el flujo unidireccional dentro del cauce de los ríos (Dudgeon et al. 2006, Nilsson et al. 2005) en los que se presentan diferentes flujos de velocidad del agua y de grandes turbulencias y forman un nuevo sistema semi-lacustre con características similares a las de un lago y con algunas influencias del río (Granado-Lorencio 2002). Estas nuevas condiciones modifican la composición y la estructura del ensamblaje de la biota acuática y en particular de la ictiofauna (Petrere 1996).

El desarrollo del sector hidroeléctrico, a nivel global, ha provocado la construcción de embalses en ríos alrededor del mundo, muchos de estos localizados a lo largo de un mismo cauce, conformando sistemas de embalses en cascada. Analizando la respuesta de algunas características del agua (v. g., temperatura, turbidez, oxígeno) así como la diversidad biótica, Ward y Standford (1983), en su planteamiento Discontinuidad Serial, definen que éstos nuevos sistemas acuáticos además de romper con el continuo del río (Vannote et al. 1980), perturban el hábitat, interrumpen el flujo de agua, de sedimentos, de nutrientes (Barbosa et al. 1999, Bunn y Arthington 2002) y de la biota acuática (Chaves-Ulloa et al. 2014); efectos que pueden ser acumulativos en sistemas que presentan múltiples embalses en serie (Stanford y Ward 2001), pues estos alteran la dinámica de nutrientes del sistema, y en consecuencia, la productividad pesquera en embalses aguas abajo (Welcomme 1985). A pesar de estos cambios, las condiciones ecológicas de los ríos tienden a restablecerse a su estado inicial, aguas abajo del sector embalsado, dado que las aguas que ingresan al río desde una cuenca superior ejercen un efecto de "restauración", puesto que oxigenan las aguas, se incrementan los sedimentos en el agua y la fauna que compone el lecho del cauce (Stanford y Ward 2001).

En Colombia, Maldonado-Ocampo et al. (2008) reportaron 213 especies para la cuenca del río Magdalena-Cauca. En embalses en Colombia, Jiménez-Segura et al. (2011a) reportaron 77 especies de peces. Entre éstas especies se encuentran nativas e introducidas, siendo éstas últimas las especies que sostienen en gran parte las pesquerías de los embalses colombianos. Dentro de la compleja red hídrica de la Cuenca Magdalena-Cauca han sido varios los ríos que suscitan interés para la generación de energía eléctrica. En el río Porce (tributario del río Nechí, afluente del río Cauca) se encuentran en operación la central Guadalupe III y IV, los embalses Miraflores-Troneras, Río Grande, Porce II y III, además de otros planteados como Porce IV y diversas microcentrales.
Desde la década de 1960 la cuenca alta del río Porce (tramo conocido como río Medellín) ha tenido grandes cambios en sus hábitats acuáticos. El mal manejo de las aguas residuales e industriales que caen al río Medellín y la pérdida de bosque para la construcción de viviendas han modificado, no solo la calidad del agua, sino también la tipología del pulso de caudal, ocasionando que su ictiofauna sea pobre en el número de especies (Uran y Torrente 2001). La carga de materia orgánica que lleva el río Porce al ingresar al embalse Porce II hace de éste un sistema hiper-eutrófico con alta productividad primaria y con bajos niveles de oxígeno (EPM 2009). La retención de esta carga orgánica dentro de éste embalse permite que el agua turbinada por la central hidroeléctrica Porce II tenga mejor calidad. El embalse Porce III recibe estas aguas, así como las que vienen de la central Guadalupe IV y ha sido definido como un sistema eutrófico (Silva 2008).

Petrere (1996) planteó que la construcción de embalses en cadena (ó cascada) en ríos altamente contaminados, aumenta la productividad biológica y mejora las condiciones del agua abajo de éstos embalses. Con base en esta hipótesis se plantearon dos preguntas de investigación sobre la respuesta de la ictiofauna a las condiciones de embalses en cascada en el río Porce: a) ¿cómo está estructurado el ensamblaje en dos embalses de cascada en el río Porce y b) ¿ésta estructura se mantiene en dirección cola-presa y en su interacción con el eje vertical dentro de cada embalse? Para responderlas, analizamos la composición de especies y la estructura del ensamblaje de peces en los embalses Porce II y Porce III, ubicados sobre el cauce principal del río Porce, durante diferentes periodos climáticos (lluvias, estiaje), entre los años 2011 y 2013.

\section{MATERIALES Y MÉTODOS}

Área de estudio. El río Porce, llamado río Medellín en los primeros $101 \mathrm{~km}$, nace en el alto San Miguel a $2660 \mathrm{~m}$ s.n.m., al oeste de la cordillera Central de los Andes. En la cuenca se observa un régimen de distribución bi-modal de las lluvias (dos periodos de máximas lluvias: marzo a junio y septiembre a noviembre, y dos de reducción: diciembre a febrero y julio a agosto). La longitud del cauce del río es de $226 \mathrm{~km}$ y desemboca en el río Nechí (afluente del río Cauca), a los 100 m s.n.m. Su caudal medio anual es de $113,4 \mathrm{~m}^{3} \cdot \mathrm{seg}^{-1}$. En el sector medio de la cuenca, el río Porce corre por un sistema de montaña de gran pendiente y por sus laderas fluyen pequeñas quebradas con caudales entre 0,01 y $3 \mathrm{~m}^{3} \cdot \mathrm{s}^{-1}$. En el año 2000 fue construido, sobre el cauce principal del río, el embalse Porce II, a los 924 m s.n.m. En el año 2011 fue construido, aguas abajo del embalse Porce II, 
el embalse Porce III, a los 680 m s.n.m. Estos embalses están localizados aguas abajo de la ciudad de Medellín, capital del departamento de Antioquia, a 120 y $147 \mathrm{~km}$ respectivamente (figura 1). El embalse Porce II se considera un embalse "hiper-eutrófico" (EPM 2009); mientras que el embalse Porce III se considera un embalse eutrófico (Silva 2008). El volumen de agua almacenado de Porce II depende de los aportes del río Porce y de la operación de la central hidroeléctrica, en tanto que el embalse Porce III depende de la interacción entre los aportes de la descarga de la central Porce II, de la central hidroeléctrica Guadalupe III y IV, del río Guadalupe, y de la operación de la central hidroeléctrica Porce III. En la tabla 1 se presentan algunas características limnológicas de los diferentes sectores dentro de los dos embalses.

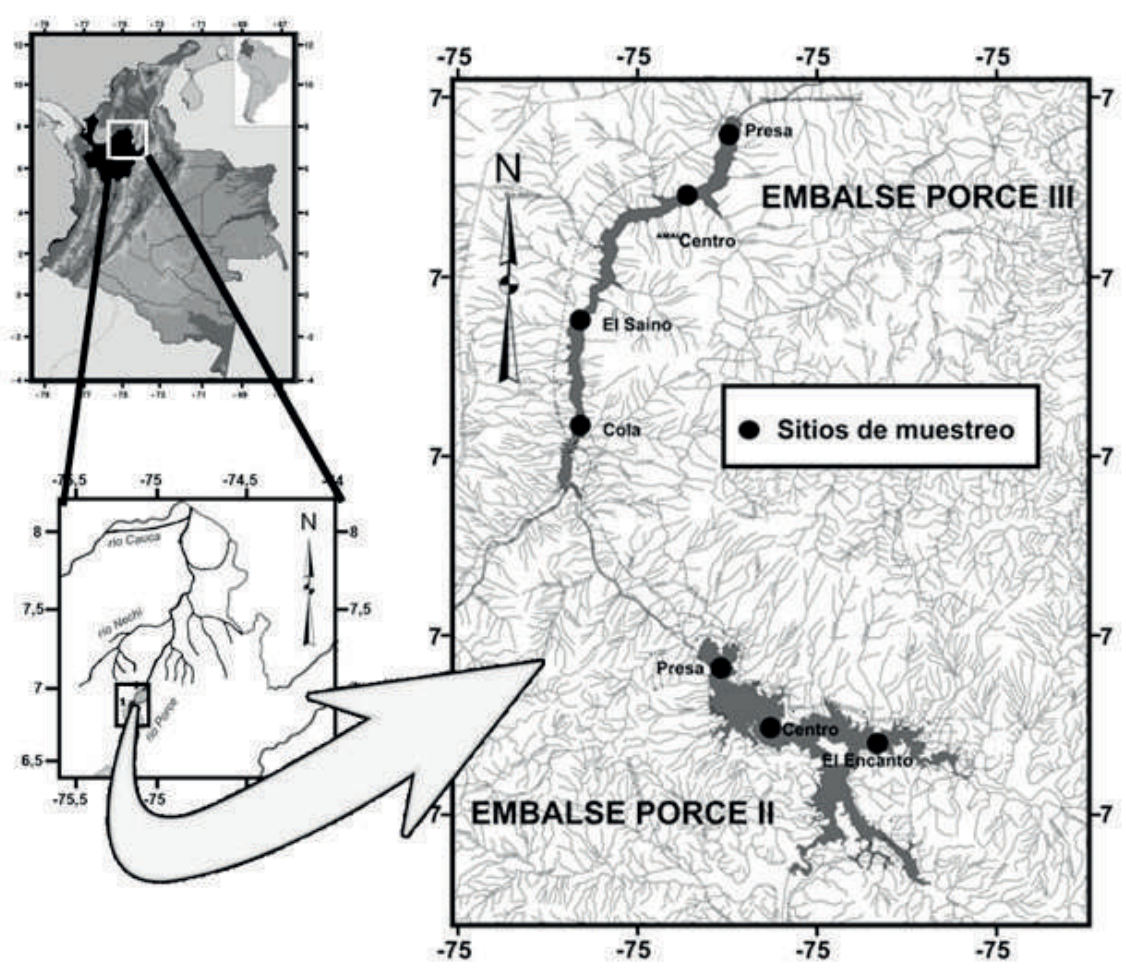

Figura 1. Ubicación Geográfica del área de estudio

Tabla 1. Características limnológicas en el gradiente longitudinal del embalse de Porce II y Porce III. Las variables limnológicas se presentan como media \pm desviación estándar; la profundidad como la media y el rango mínimo y máximo

\begin{tabular}{lcccccccc}
\hline & & \multicolumn{3}{c}{ Embalse Porce II } & \multicolumn{4}{c}{ Embalse Porce III } \\
\cline { 3 - 9 } Variable & $\begin{array}{c}\text { Eje } \\
\text { vertical }\end{array}$ & Cola & Centro & Presa & Cola & $\begin{array}{c}\text { Centro-El } \\
\text { Saino }\end{array}$ & Centro & Presa \\
\hline & & & & & & & & \\
\hline
\end{tabular}




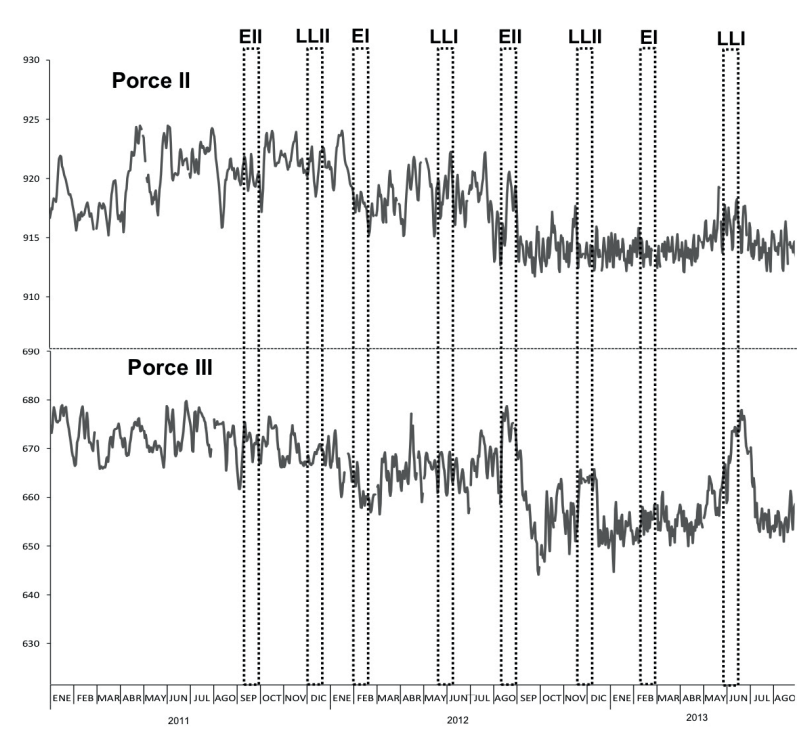

Figura 2. Nivel diario del agua de los embalses Porce II y Porce III. Los valores en el eje $X$ corresponden a la altura sobre el nivel del mar (m s.n.m.). Datos de las Empresas Públicas de Medellín. El: Estiaje I, Ell: Estiaje II, LLI: Lluvias I, LLII: Lluvias II.

Diseño muestral. Entre los años 2011 y 2013, se realizaron ocho muestreos en periodos pluviométricos contrastantes (lluvias y estiajes). Para verificar la hipótesis asociada con el cambio que imprimen los dos embalses a las características del ensamblaje a lo largo del eje longitudinal, las muestras se tomaron en la zona litoral de los diferentes sectores dentro del embalse. Dentro de cada embalse, los sitios de muestreo se localizaron siguiendo el modelo general de Thornton (1990), donde se separan tres sectores principales sobre el eje longitudinal del embalse: desde la cola del embalse donde ingresa el río Porce (cola o zona ribereña), el centro del embalse (zona de transición) y en dirección hacia el muro de la presa (zona lacustre o lagunar) (figura 2). Para incorporar el efecto de la profundidad en la estructura del ensamblaje de especies, en cada sector se tomaron muestras a $0.5 \mathrm{~m}$ y $3 \mathrm{~m}$ de la superficie (superficie) y de $4 \mathrm{~m}$ a $7 \mathrm{~m}$ de la superficie (fondo).

Las capturas se realizaron entre las 07:00 y 14:00 horas. Se utilizaron redes de espera (100 m longitud, $3 \mathrm{~m}$ de alto) con diferentes tamaños de malla $(0,01$ a $0,15 \mathrm{~m})$. Se conservó el esfuerzo de pesca ( 6 horas. $300 \mathrm{~m}^{-2} \cdot \operatorname{Red}^{-1}$ ) entre los sitios de muestreo para permitir comparaciones. En cada sitio del embalse se emplearon tres redes, dos superficiales (a $0,5 \mathrm{~m}$ de la superficie) y una de fondo (a $4 \mathrm{~m}$ de la superficie).
En campo, los ejemplares fueron identificados y su información (especie, longitud estándar, peso) fue registrada bajo un código. Aquellos ejemplares de difícil identificación en campo fueron anestesiados por diez minutos por inmersión en solución de Eugenol (aceite de clavo, sedante usado en peces; Neiffer y Stamper, 2009), se fijaron en solución de formalina al 10\% (Murphy et al. 1996) y se almacenaron en bolsas plásticas debidamente etiquetadas con la información de campo (sitio, hora y fecha de captura). A cada ejemplar se le determinó su especie con la ayuda de claves taxonómicas apropiadas a nivel de familia y género. La identidad taxonómica fue verificada en Eschmeyer y Fong (2017).

Análisis de datos. Se definió el número de especies, así como la abundancia numérica de cada especie dentro de la muestra. Para definir la categoría de cada especie dentro del ensamblaje (dominante, ocasional, común, rara) se utilizó su frecuencia de ocurrencia en las muestras obtenidas, así como su abundancia numérica (Sokal y Rolfh 1995). Para construir las gráficas de dispersión en $2 \mathrm{D}$ se empleó el software SimagPlot 12.0.

Para estimar los valores de diversidad alfa (Fisher 1943) por sector y periodo pluviométrico y la dominancia (Whittaker 1972) se usaron las abundancias numéricas de las especies de peces. Para su cálculo se usó el software PAleontological STatistics (PAST) vers. 2.16 (Hammer et al. 2001). Dado que los datos no cumplieron con los supuestos de homogeneidad de varianzas ni normalidad, se usó una prueba de comparación de rangos (ANOVA no paramétrico, Kruskall-Wallis) para verificar si existían diferencias estadísticamente significativas en los valores de diversidad alfa, dominancia y número de especies entre los sectores de cada uno de los embalses (cola, transición, presa) y una prueba de contingencia (chi-cuadrado de Pearson) para determinar si había diferencias significativas en la interacción eje vertical (superficie y fondo) y hábitat de los embalses.

\section{RESULTADOS}

Se capturaron 16207 individuos (89,95\% en el embalse Porce II y $10,1 \%$ en el embalse Porce III) representantes de 15 especies (tabla 2). En términos de la distribución porcentual de la abundancia numérica el orden Characiformes agrupó el 40\%, el Perciformes 33,33\%, Siluriformes 13,33\% y Cyprinodontiformes 13,33\%. En los embalses Porce II y Porce III las especies dominantes dentro del ensamblaje fueron Astyanax microlepis, Andinoacara latifrons, Roeboides dayi y Coptodon rendalli; Brycon henni solo 
Tabla 2. Distribución de la abundancia relativa por especie en los embalses Porce II y Porce III. *Especies no nativas

\begin{tabular}{ccc}
\hline \multirow{3}{*}{ Taxa } & \multicolumn{2}{c}{$\begin{array}{c}\text { Abundancia } \\
\text { relativa (\%) }\end{array}$} \\
\cline { 2 - 3 } & $\begin{array}{c}\text { Porce } \\
\text { II Porce }\end{array}$ & III \\
\hline
\end{tabular}

\section{CHARACIFORMES}

Anostomidae

Leporellus vittatus

(Valenciennes1850)

0,18

Characidae

$\begin{array}{lll}\text { Roeboides dayi (Steindachner1878) } & 2,99 & 7,92 \\ \begin{array}{l}\text { Hyphessobrycon proteus } \\ \text { Eigenmann1913 }\end{array} & 0,11\end{array}$

Astyanax microlepis Eigenmann1913 78,63 58,26

Bryconidae

Brycon henni Eigenmann1913 $\quad 0,01 \quad 16,64$

SILURIFORMES

Callichthyidae

Hoplosternum magdalenae

Eigenmann1913

12,05

Loricariidae

Chaetostoma fischeri

Steindachner1879

CYPRINODONTIFORMES

Poeciliidae

$\begin{array}{lll}\text { Poecilia caucana(Steindachner1880) } & 0,11 & 0,06 \\ \text { Poecilia reticulata Peters1859* } & 0,01 & \end{array}$

PERCIFORMES

Cichlidae

\begin{tabular}{lcc}
$\begin{array}{l}\text { Oreochromis mossambicus } \\
\text { (Peters1852)* }\end{array}$ & 0,04 & \\
\hline $\begin{array}{l}\text { Oreochromis niloticus } \\
\text { (Linnaeus1758)* }^{*}\end{array}$ & 0,29 & 0,92 \\
\hline $\begin{array}{l}\text { Coptodon rendalli (Boulenger1897)* } \\
\text { Andinoacara latifrons }\end{array}$ & 2,41 & 8,53 \\
\hline $\begin{array}{l}\text { (Steindachner1878) } \\
\text { Parachromis loisellei (Bussing1989)* }\end{array}$ & 0,01 & 7,43 \\
\hline
\end{tabular}

fue dominante en Porce III mientras que Hoplosternum magdalenae lo fue en Porce II (figura 3). Estas seis especies reunieron el 99,36\% de la abundancia de los individuos capturados en los dos embalses (tabla 2). Las familias con mayor número de especies fueron Cichlidae y Characidae (cinco y cuatro especies, respectivamente). Por su parte, las familias Anostomidae, Bryconidae y Callichthyidae estuvieron representadas solo por una especie.

El número, la composición y la dominancia de especies fue diferente entre los embalses y entre los diferentes sitios de los embalses. En el embalse Porce II se capturaron 12 especies de peces, mientras que en el embalse Porce III se capturaron 10. La mayor abundancia numérica se registró en el embalse Porce II en el sector próximo a la influencia del río Porce (cola); A. microlepis, H. magdalenae y $R$. dayi agruparon el $95 \%$ de la abundancia en este embalse, en tanto que en los sitios de transición (centro) y presa del embalse, C. rendalli y A. latifrons fueron las especies más importantes en número (figura 4). Por su parte, en el embalse Porce III, A. microlepis fue la especie más abundante en todos los sectores del embalse, $B$. henni y $R$. dayi lo fueron en el sector de la cola, y $C$. rendalli y $A$. latifrons lo fueron en el sector de la presa. En todos los sectores de los embalses Porce II y Porce III se encontraron especies introducidas como C. rendalli, Oreochromis niloticus, Oreochromis mossambicus y Parachromis loisellei (tabla 2).

En el embalse Porce II, la dominancia de especies fue alta en los sectores cercanos a la entrada del río Porce, donde A. microlepis y H. magdalenae dominaron la estructura del ensamblaje; mientras que en los sitios cercanos al muro del embalse (centro y presa) los valores de dominancia disminuyeron y el ensamblaje estuvo conformado por las especies $H$. magdalenae, A. latifrons y C. rendalli (tabla 2 , figura 4). Desde el sector medio (centro) hasta el sector presa del embalse Porce III, la estructura estuvo dominada por las especies A. microlepis, B. henni y C. rendalli. Éstas especies y otras como $R$. dayi y A. latifrons conformaron el ensamblaje encontrado en el sector cola.

En la interacción sector-profundidad de los embalses Porce II y Porce III, no se observaron diferencias significativas (Porce II $p=0,19$; Porce III $p=0,32$ ). A pesar de este resultado es evidente la reducción en la abundancia, en la diversidad y en el número de especies en el eje longitudinal (cola, centro, presa) y vertical (superficie, fondo) dentro del embalse Porce II (tabla 3). En el embalse Porce III, el sector cola (cerca al río) y presa presentó el mayor número de especies en la superficie y en los otros (saino y centro), en el fondo (tabla 3, figura 1). 

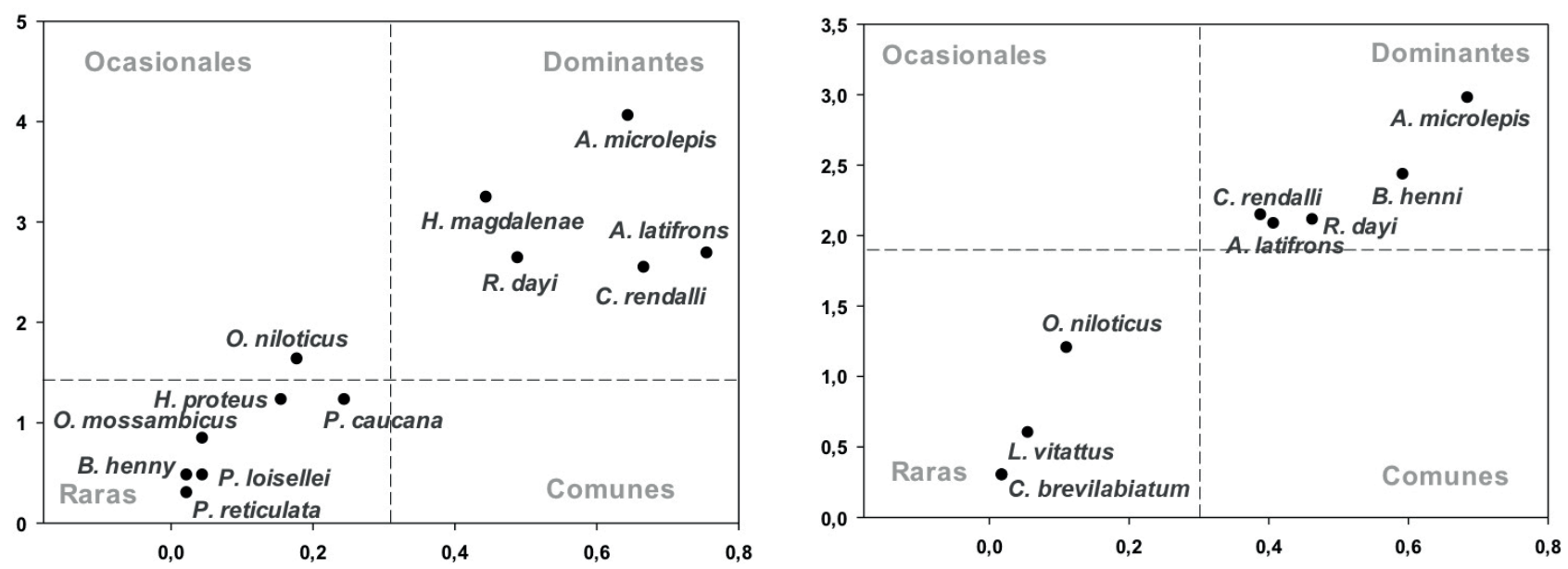

Figura 3. Ensamblaje de especies de peces en los sistemas de embalse de la cuenca media del río Porce. A. Embalse Porce II, B. Embalse Porce III. Eje vertical: Abundancia (Log); Eje Horizontal: Frecuencia de Ocurrencia Relativa. Parámetros en el eje $Y$ : mediana de la abundancia. Parámetros en el eje $X$ : media de la frecuencia.

\section{Cola (El Encanto)}

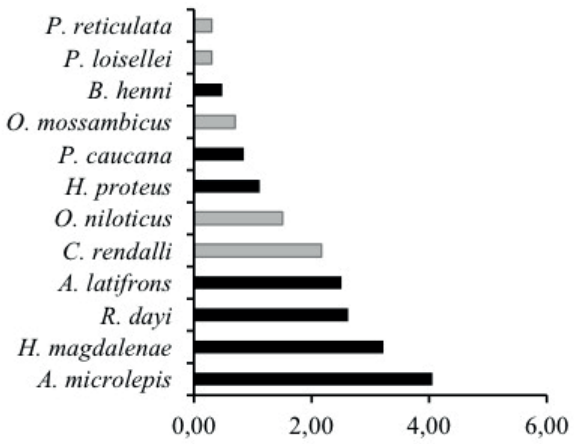

Transición (Centro)

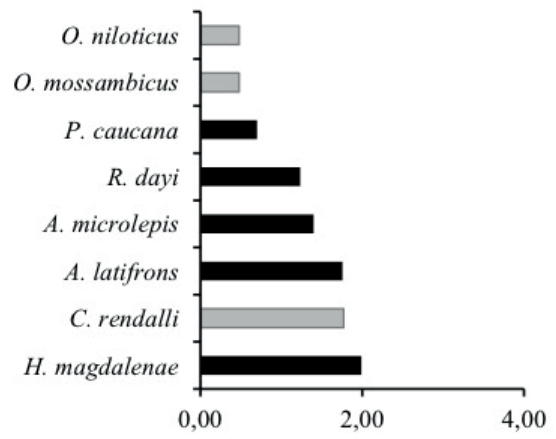

Presa

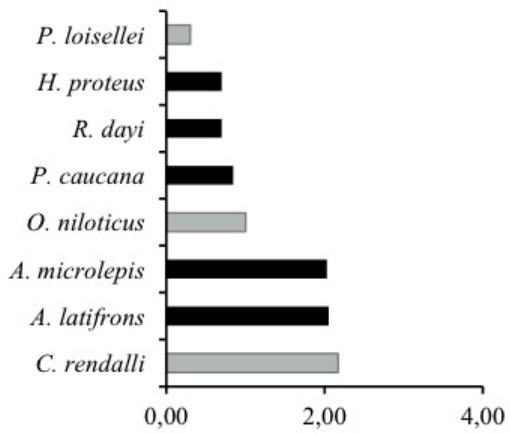

Cola

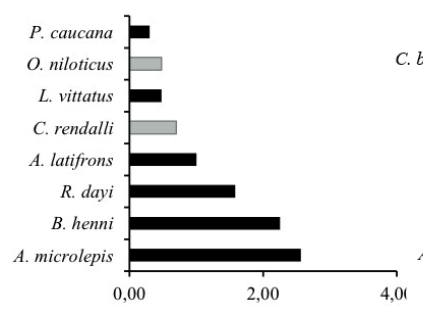

Transición (EI Saino)

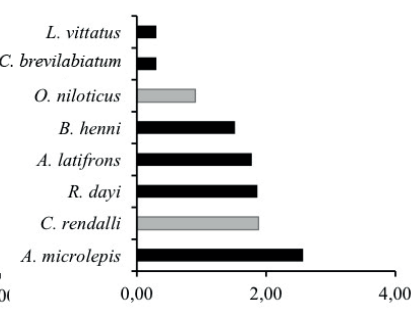

Transición (Centro)

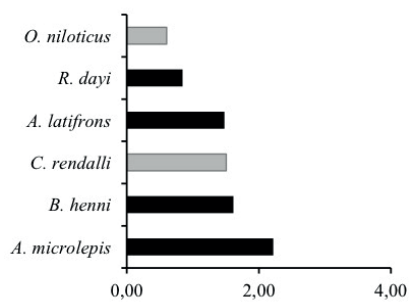

Presa

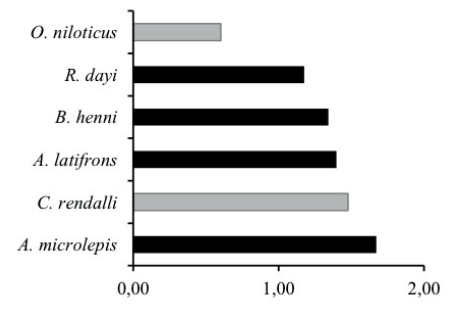

Figura 4. Distribución de la abundancia numérica relativa de las especies de peces dentro de los embalses del río Porce. A. Embalse Porce II, B. Embalse Porce III. Barras en color gris pertenecen a especies introducidas.

En el sector cola y saino, la dominancia fue mayor en el fondo (dominando A. microlepis, B. henni y $R$. dayi), en tanto que en el sector de la presa la dominancia fue mayor en la zona superficial (dominando C. rendalli, O. niloticus y B. henni). El mayor número de individuos se obtuvo en los sectores cola, saino y centro del embalse. 
Tabla 3. Valores medios y rangos de algunos descriptores del ensamblaje de especies de peces en los embalses del río Porce. Media (Diversidad Alfa y Dominancia), mediana (número de especies). Diferencias significativas $(p<0,05)$ entre: *los hábitats, ${ }^{\S}$ Entre periodos pluviométricos

\begin{tabular}{|c|c|c|c|c|c|c|}
\hline \multirow[t]{2}{*}{ Sistema } & \multicolumn{2}{|c|}{ Sector } & $\begin{array}{l}\text { Número } \\
\text { de }\end{array}$ & Diversidad a & Dominancia & Abundancia \\
\hline & \multicolumn{2}{|l|}{ General } & $4(2-11)^{*}$ & $1,62(0,59-5,45)^{\S}$ & $0,53(0,21-0,82)$ & $4,40(1,40-8,47)^{*}$ \\
\hline \multirow{6}{*}{ Porce II } & \multirow{2}{*}{ Cola } & Superficie & $6(3-11)$ & $1,59(0,76-5,45)$ & $0,62(0,38-0,82)$ & $2,61(0,70-3,47)$ \\
\hline & & Fondo & $5(1-7)$ & $0,85(0,45-1,06)$ & $0,78(0,54-1)$ & $2,31(0,48-3,27)$ \\
\hline & \multirow{2}{*}{ Centro } & Superficie & $3(1-6)$ & $1,57(0-2,76)$ & $0,50(0,28-1)$ & $1,14(0,30-1,81)$ \\
\hline & & Fondo & $1,5(1-6)$ & $0,83(0-2,50)$ & $0,78(0,30-1)$ & $0,82(0,30-1,49)$ \\
\hline & \multirow{2}{*}{ Presa } & Superficie & $3(2-5)$ & $2,25(0,40-9,28)$ & $0,49(0,28-0,79)$ & $1,38(0,78-1,91)$ \\
\hline & & Fondo & $1(0-4)$ & $0,23(0-1,00)$ & $0,49(0-1)$ & $0,54(0,00-1,96)$ \\
\hline \multirow{9}{*}{ Porce III } & \multicolumn{2}{|l|}{ General } & $4(0-6)$ & $1,05(0-2,26)^{\S}$ & $0,48(0-1)$ & $1,42(0-2,26)^{*}$ \\
\hline & \multirow{2}{*}{ Cola } & Superficie & $6(0-7)$ & $1,87(0-2,71)$ & $0,36(0-0,63)$ & $1,40(0-1,82)$ \\
\hline & & Fondo & $5(0-8)$ & $1,45(0-3,32)$ & $0,43(0-0,83)$ & $1,71(0-2,22)$ \\
\hline & \multirow{2}{*}{ Transición-Saino } & Superficie & $5(4-7)$ & $1,66(1,27-2,05)$ & $0,38(0,26-0,65)$ & $1,51(1,20-1,79)$ \\
\hline & & Fondo & $5(4-7)$ & $1,44(0,94-2,06)$ & $0,44(0,32-0,85)$ & $1,63(1,43-2,29)$ \\
\hline & \multirow{2}{*}{ Centro } & Superficie & $2(1-3)$ & $1,08(0-2,62)$ & $0,57(0,43-1)$ & $0,65(0,48-1,36)$ \\
\hline & & Fondo & $2(0-4)$ & $0,60(0-1,74)$ & $0,60(0-1)$ & $0,95(0-2,17)$ \\
\hline & \multirow{2}{*}{ Presa } & Superficie & $4(0-5)$ & $3,02(0-9,28)$ & $0,38(0-0,63)$ & $0,93(0-1,42)$ \\
\hline & & Fondo & $2,5(0-6)$ & $1,54(0-5,45)$ & $0,27(0-0,65)$ & $0,65(0-1,60)$ \\
\hline
\end{tabular}
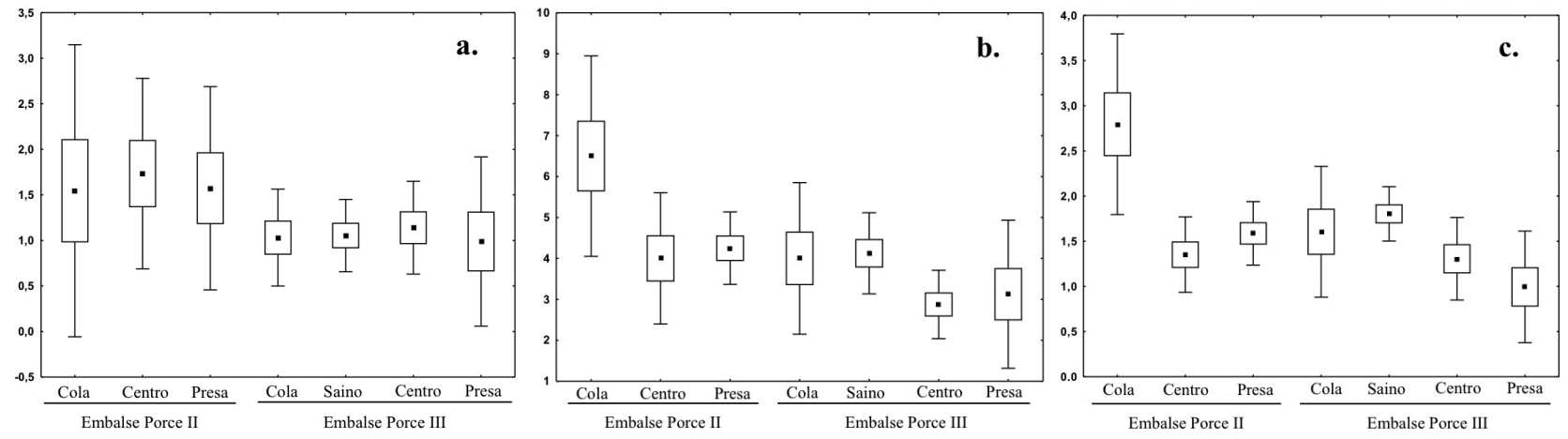

Figura 5. Características emergentes de los ensamblajes de peces a lo largo del eje longitudinal de los embalses de Porce II y Porce III del río Porce. A. Diversidad Alfa. B. Número de especies. C. Abundancia relativa (Log 10 ). Punto: media, Caja: error estándar, barras: desviación estándar. 

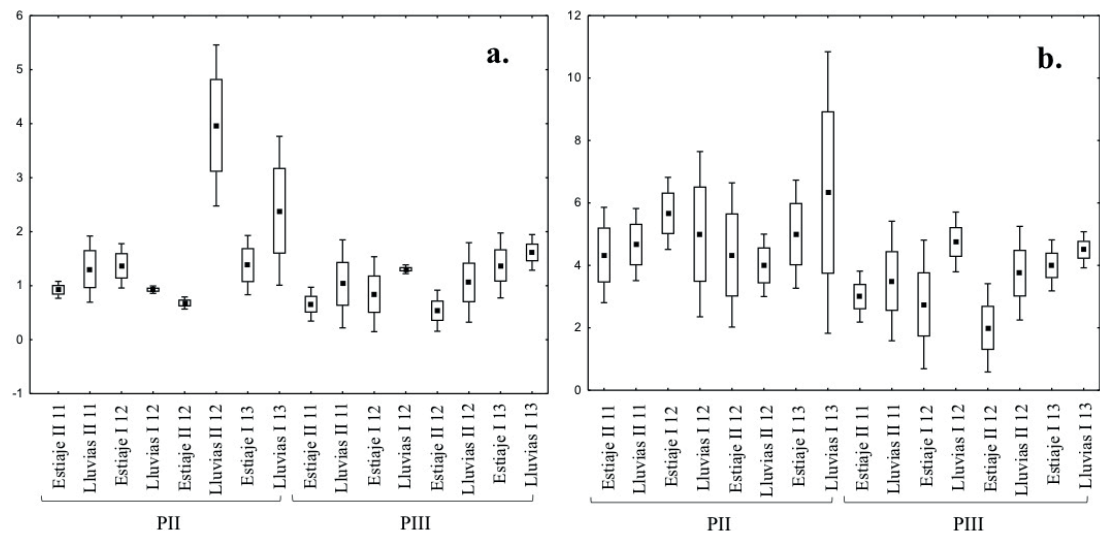

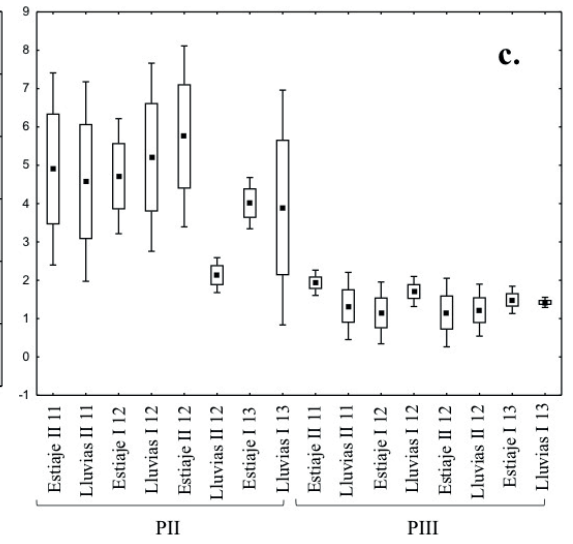

Figura 6. Características emergentes de los ensamblajes de peces en la escala temporal entre los años 2011 y 2013. A. Diversidad alfa. B. Número de especies. C. Abundancia relativa $\left(\log _{10}\right)$. Punto: media, Caja: error estándar, Barras: Desviación estándar. I: primer semestre del año, II: segundo semestre del año; años: 2011 (11), 2012(12) ,2013(13). PII: embalse Porce II y PIII: embalse Porce III.

El número de especies presentó diferencias significativas entre los sectores solo dentro del embalse Porce II $\left(\mathrm{H}_{2,24}=\right.$ $6,15 ; p<0,05)$, y la abundancia numérica fue diferente entre los dos embalses y dentro de cada embalse (Porce II, $\mathrm{H}_{2,24}$ $=8,65, p<0,05 ;$ Porce III, $\mathrm{H}_{3,32}=10,50, p<0,05$ ) (figura 5).

Aunque la diversidad alfa no presentó diferencias significativas $(p<0,05)$ entre los sectores dentro de cada uno de los embalses (tabla 3), si se observaron algunas tendencias durante los diferentes momentos de muestreo. En el embalse Porce III, la diversidad fue menor que en Porce II y no tuvo grandes variaciones entre sectores. Dentro del embalse Porce II, la mayor desviación en los valores de la diversidad fue en sector de la cola (ingreso del río Porce), mientras que en Porce III la mayor diversidad se encontró en el centro del embalse y las mayores desviaciones en el sector presa.

La abundancia numérica fue mayor en el embalse Porce II que en Porce III y no se observaron diferencias entre los periodos de lluvias (figura 6), así como en el número de especies y la dominancia $(p<0,05)$ (tabla 3). En la dimensión temporal la diversidad alfa presentó diferencias significativas. En el embalse Porce III, los mayores valores se registraron en las temporadas de lluvias $\left(\mathrm{H}_{7,32}=13,96, p<0,05\right)$; mientras que en el embalse Porce II solo se presentaron en el periodo de lluvias de 2012 y $2013\left(\mathrm{H}_{7,24}=16,73, p<0,05\right)$ (figura 6).

\section{DISCUSIÓN}

El ensamblaje de especies en el sector del cauce del río Porce que fue embalsado, es pobre en especies.
Jiménez-Segura et al. (2013) estimaron que dentro de este rango altitudinal ( $680-924$ m s.n.m.) se espera encontrar aproximadamente 81 especies en sistemas de ríos naturales, y entre 2 y 31 especies en embalses de la cuenca Magdalena-Cauca y la vertiente del Pacífico (Jiménez-Segura et al. 2011a). Agostinho (1999) afirma que la comunidad de peces en los embalses resulta de la re-estructuración de la comunidad de peces original del río, y la composición que se observa también dependerá del tiempo de formación del embalse, así como de sus características (forma, profundidad, tiempo de retención, área de espejo de agua).

Dentro de la nueva estructura del ensamblaje, especies como A. microlepis, H. magdalenae, $R$. dayi y $C$. rendalli fueron dominantes en el ensamblaje de los embalses Porce II y Porce III. Estas especies son comunes (poco abundantes, muy frecuentes) en lagos del plano inundable del río Magdalena (Granado-Lorencio et al. 2012) y raras o ausentes en sistemas ribereños (Jiménez-Segura et al. 2014). Aunque no se cuenta con información completa sobre las estrategias de vida de estas especies en nuestro país, Gomes y Miranda (2001) afirman que algunas estrategias de vida permiten que ciertas especies nativas sean exitosas en sistemas de flujo de agua lento: a) tamaños de camada y cuidado parental, b) uso de un reducido número de compartimentos dentro de la red trófica (v. g., herbívoros, planctófagos, detritívoros) y ciclos de vida rápidos. Entonces, si estas especies se encontraban dentro del ensamblaje ribereño original, es posible que la alta fecundidad sin cuidado parental, como en el caso de $A$. microlepis y $R$. dayi (Loaiza y Jiménez-Segura 2013) o 
la baja fecundidad con cuidado parental, en el caso de $H$. magdalenae y $C$. rendalli (Londoño y Jiménez-Segura 2013), así como su dieta (v. g., herbivoría, omnivoría) (Universidad de Antioquia y EPM 2013), sea la causa de su éxito en los embalses Porce II y Porce III.

El embalse Porce II presentó un mayor número de especies y mayores abundancias comparado con Porce III; el ensamblaje se caracterizó por la ausencia de especies típicas de este sector del río Porce como B. henni, $C$. brevilabiatum y L. vittatus y la presencia de especies introducidas como $C$. rendalli, $P$. loisellei y $O$. niloticus, en tanto que en el embalse Porce III aún persisten estas especies y las especies introducidas son menos abundantes. Esta estructura de la ictiofauna en los dos embalses es resultado de las características propias a cada embalse y que son determinantes en la persistencia de las poblaciones de estas especies dentro de estos cuerpos de agua. El embalse Porce II tiene 12 años de formado, recibe la descarga de materia orgánica de Medellín (Antioquia), su forma dendrítica con numerosas bahías internas puede favorecer la oferta de hábitats, la temporalidad de ingreso de agua depende de la estacionalidad climática de la cuenca aportante, es un sistema más estable que el embalse Porce III pues su tiempo de retención hidráulico es de 43 días y tienen un área de espejo de agua mucho mayor $(13,78$ $\mathrm{km}^{2}$, cota mínima de inundación). En tanto que el embalse Porce III es un sistema joven con apenas cuatro años de formación, poco complejo en su estructura (forma alargada, menor desarrollo de borde, menor área de inundación, 4,61 $\mathrm{km}^{2}$ ), con fuertes oscilaciones en el volumen de agua, pues su condición geomorfológica le permite ser menos estable, sus entradas dependen de la operación de las centrales Guadalupe III y IV y Porce II y su tiempo de retención es de ocho días. La persistencia de las poblaciones nativas en el embalse Porce III en los próximos años dependerá de las condiciones que le sean favorables a su reclutamiento. La respuesta de estas especies a esta nueva dinámica hidrológica dentro de éste sector en el río Porce debe ser objeto de estudio en un futuro cercano.

Son diversos los autores que han encontrado resultados similares en otras regiones de Suramérica. AraujoLima et al. (1995), encontraron que el área del embalse influye en la cantidad de especies; Agostinho (1999, 2007) y Petrere (1996) mencionaron que la riqueza decrece con la edad del embalse, y Gutiérrez-Bonilla y Álvarez-León (2011), Jiménez-Segura et al. (2011a) y Larrahondo (1993), indicaron que los embalses son sistemas acuáticos que favorecen el crecimiento de las poblaciones de especies exóticas, las cuales se convierten en el sustento de la actividad pesquera artesanal. Sin embargo, al considerar embalses en cascada, estas tendencias identificadas no necesariamente mantienen estos patrones. Aunque el tiempo de retención del agua pueden influir en la producción de plancton y carga de nutrientes en los embalses, la proximidad geográfica entre dos presas también es influyente (Barbosa et al. 1999), y en última instancia, puede reducir la abundancia numérica de peces dentro del sistema (Draštíket et al. 2008, Jackson y Marmulla 2001). No obstante, con la edad del embalse la riqueza disminuye (Agostinho et al. 2007) y el rendimiento pesquero tiende a incrementar (JiménezSegura et al. 2011a). Así que, es probable que el bajo número de ejemplares capturados en el embalse Porce III corresponda al tiempo de vida de este y a la interacción entre los embalses en cascadas. Esto ya había sido analizado en Petrere (1996) quien afirmó que, en cuencas con problemas de contaminación, la calidad del agua en aquellos embalses localizados aguas abajo de otros embalses tendrá mejores condiciones para la ictiofauna dado que los embalses aguas arriba actuarán como sistemas filtro. El embalse de Porce II, podría actuar como filtro de nutrientes y sedimentos y así cambiarían las condiciones físico-químicas del agua que entra al embalse Porce III ( v. g., menor carga de sedimentos y de nutrientes). Estas condiciones podrían ser favorables a la ictiofauna; sin embargo, el embalse Porce III presenta menor número de especies, así como menor abundancia de individuos. Entonces, se debe considerar que si bien, la calidad del agua puede ser diferente a la del embalse Porce II, las condiciones propias al embalse como son su edad, su estructura física similar a la de un río y las grandes oscilaciones en el volumen almacenado debido a la operación de la central y al ingreso del caudal turbinado por las centrales hidroeléctricas presentes aguas arriba (Porce II, Guadalupe III y IV), puedan ser parte de la razón por la que la ictiofauna del embalse Porce III si es comparada con la de Porce II es poco diversa y poco abundante.

La composición y abundancia numérica de la ictiofauna cambió a lo largo del eje longitudinal (cola-presa) de los embalses. De acuerdo con Margalef (1983), los embalses son sistemas híbridos entre un río y un lago, los cuales están biológica, física y químicamente estructurados desde la cola hasta la presa. Los cambios involucran estratificación en la columna del agua y velocidad en el flujo del agua, esto asociado a la forma del hábitat y al aporte de sedimentos al sistema. Agostinho et al. (2007) indican que las especies nativas del ensamblaje original tienen mayor oportunidad de persistir en aquellos sectores del embalse cercanos a la influencia del río; estas especies en su mayoría son de pequeño a mediano porte, omnívoras y menos exigentes a las condiciones físicas y químicas del embalse para su reproducción y reclutamiento. En los embalses analizados 
especies como A. microlepis, H. magdalenae y $R$. dayi fueron más abundantes en la cola del embalse Porce II mientras que en la presa son más abundantes especies de Ciclidos (C. rendalli, A. latifrons), en tanto que en la cola de Porce III lo fue A. microlepis, R. dayi y B. henni, y en la presa fue $A$. microlepis y $A$. latifrons.

La presencia e importancia de $B$. henni en el embalse Porce III sugiere que la especie puede estar aprovechando los recursos que circulan en el nuevo sistema acuático. La formación del embalse Porce III permite ahora a los individuos de la especie salir de sus tributarios y utilizar el embalse como ruta de movimiento. Su capacidad de remontar cuencas de alta pendiente y hacer movimientos cortos entre cuencas (Builes y Lara 1980, Builes y Uran 1974) le ha permitido a la especie ocupar los cuerpos de agua del embalse Porce III. Es posible que a medida que el tiempo pase, la abundancia numérica de la especie se reduzca restringiéndose nuevamente a las quebradas. Aunque esta es una hipótesis que debe verificarse a futuro.

La carga de nutrientes y sedimentos que ingresa a los embalses, así como la temperatura del agua son factores que pueden influir en la estructura del ensamblaje en el eje longitudinal (dirección cola-presa) y vertical (superficie-fondo). En el embalse Porce II se detectó estratificación vertical (independientemente del sector) en las características del ensamblaje de especies. De acuerdo con Umaña (2006), la estratificación (epipelágica y batipelágica) de la columna del agua, depende del arrastre que ejerce la entrada del río, la salida de agua y la forma misma del embalse de manera que la estratificación ocurre en los sitios más profundos, cercanos a la presa. Es posible que la diferencia en la carga de sedimentos que reciben los embalses Porce II (río Porce) y Porce III (descarga del Porce II y río Guadalupe), así como la variabilidad en el nivel del agua (debido a los tiempos de retención), son variables que influyen en la estructura de la ictiofauna tanto en el eje longitudinal (cola-presa) como en el vertical (superficie y fondo). El embalse Porce II actúa como trampa de sedimentos, así que la transparencia del agua será mayor en el embalse Porce III. La alta carga de sedimentos que le aporta el río Porce al embalse Porce II genera condiciones bajas en oxígeno en el fondo de este cuerpo de agua, influyendo en que el ensamblaje de especies en la región epipelágica sea más rico en especies y diverso, independientemente del sector del embalse. En tanto que, en el embalse Porce III, la alta variabilidad en el nivel del agua, puede ser un factor causante de la baja abundancia de individuos, especialmente para aquellas especies que anidan y se refugian en la ribera del embalse (v. g., O. niloticus y C. rendalli), los cambios abruptos en el nivel del agua, deja en evidencia sus áreas de anidamiento, impidiendo el éxito desarrollo de sus progenies.

Brycon henni se encontró entre los diferentes hábitats del embalse Porce III, presentando mayor preferencia por las áreas próximas a la influencia del río. Varios autores (Magallanes y Tabares 1999, Montoya-López et al. 2006, Trahl 1973) mencionan que esta especie puede habitar tanto en sistemas lóticos como lénticos; de hecho, forma parte del ensamblaje de especies en ambientes cenagosos del río Magdalena (Jiménez-Segura et al. 2011b). Su hábito trófico omnívoro puede ser parte de la razón que le permite ocupar diferentes ambientes independientemente de la calidad en la oferta de alimento. Agostinho et al. (1999) afirma que las especies con hábitos tróficos generalistas son las más exitosas en los embalses puesto que pueden explorar los diferentes recursos que se ofrecen.

La diversidad en los dos embalses estuvo influenciada por las temporadas de lluvias. No obstante, esto podría estar más asociado con los bajos niveles del agua encontrados en los embalses que por la temporalidad climática (figura 2), pues la mayor abundancia de individuos y el número de especies se registraron en estos periodos (figura 6 B y 6 C). Esta misma tendencia se observó en los embalses de Betania y Urra I, donde las mayores capturas se registraron durante los menores niveles del agua (Jiménez-Segura et al. 2011a). De acuerdo con Agostinho (2007) y Pompeu y Godinho (2006), en aquellos embalses construidos en cascada, la temporalidad climática no influye en la estructuración del ensamblaje de especies de peces dentro del embalse, así como sucede en un ambiente ribereño natural. La pérdida de conectividad con sistemas acuáticos localizados aguas abajo, elimina la posibilidad de ingreso de nuevas especies al ensamblaje de especies dentro del embalse (Ward y Stanford 1995). A pesar que el nivel de los dos embalses no está definido por la temporalidad climática local, es posible que, durante las lluvias torrenciales, los peces sin estructuras corporales que les permitan fijarse al sustrato presente en las quebradas tributarias a los dos embalses sean arrastrados hacía el cuerpo principal del embalse. En general, las condiciones en los embalses Porce II y Porce III son favorables a las poblaciones de las especies que como A. microlepis, $R$. dayi y $H$. magdalenae, se reproducen independientemente de la estacionalidad climática (Loaiza 2013, Londoño 2013) y dominan en el ensamblaje de especies. La hipótesis que las lluvias torrenciales pueden generar movimientos locales entre las quebradas y los embalses debe ser validada en futuros estudios.

En conclusión, la formación de embalses en cascada genera cambios en la composición, riqueza y abundancia 
numérica de la fauna íctica; condiciones a las que se ajusta el ensamblaje de especies de peces conformando así ensamblajes propios a cada hábitat del sistema. No necesariamente los embalses ubicados aguas abajo dentro de cadenas de embalses a lo largo del cauce ofrecen mejores condiciones para la biota acuática tal y como se plantea en Petrere (1996). La profundidad y la morfología del embalse en interacción con temporalidad climática pueden ser definitivas en la estructuración del ensamblaje dentro de estos cuerpos de agua. La respuesta de la ictiofauna en la construcción progresiva de embalses en cascada no solo dependerá de la conformación propia de la cuenca aportante, sino de las características y dinámicas del embalse, así como de las condiciones físicas y químicas de los cuerpos de agua.

\section{AGRADECIMIENTOS}

Esta investigación se realizó dentro del convenio de cooperación No. 2011-000558 entre Empresas Públicas de Medellín (EPM) y la Universidad de Antioquia. Al señor Diego Cataño por su colaboración durante la toma de datos en campo, a los funcionarios de Empresas Públicas de Medellín (lancheros, administradores de embalse) que nos acompañaron y apoyaron en el trabajo de campo, a todo el equipo de investigadores que conforman el Grupo de Ictiología de la Universidad de Antioquia por sus valiosos aportes, comentarios y acompañamiento durante el trabajo de investigación. Y a José Álvarez y Helena Cagliano por la traducción y revisión del resumen en ingles.

\section{REFERENCIAS}

Agostinho AA, Miranda LE, Bini LM, Gomes LC, Thomaz SM, Suzuki HI. 1999. Patterns of Colonization in Neotropical Reservoirs and Prognoseson Aging. En: JG Tundisi, M Straškraba editores. Theoretical reservoir ecology and its applications. International Institute of Ecology, Brazilian Academy of Sciences, Backhuys Publishers. p. 227-265.

Agostinho AA, Gomes LC, Pelicice FM. 2007. Ecologia e manejo de recursos pesqueiros em Reservatórios do Brasil. Maringá (Paraná): Editora da Universidade Estadual de Maringá.

Agostinho AA, Pelicice FM, Gomes LC. 2008. Dams and the fish fauna of the Neotropical region: impacts and management related to diversity and fisheries. Brazilian Journal of Biology, 68(4): 1119-32. http://dx.doi.org/10.1590/S1519-69842008000500019

Araujo-Lima CARM, Agostinho AA, Fabré N. 1995. Trophic aspects of fish communities in Brazilian rivers and reservoirs. En: JG Tundisi, CEM Bicudo, T Matsamura-Tundisi editores. Limnology in Brazil. Rio Janeiro (Brasil): Brazilian Academy of Sciences. p. 105-135.
Barbosa FAR, Padisak J, Espindola ELG, Borics G, Rocha O. 1999. The cascading reservoir continuum concept (CRCC) and its application to the River Tietê-Basin, São Paulo State, Brazil. En: JG Tundisi, M Straškraba editores. Theoretical reservoir ecology and its applications. International Institute of Ecology, Brazilian Academy of Sciences, Backhuys Publishers. p. 425-437.

Builes J, Lara L. 1980. Informe sobre las actividades realizadas durante el año 1980 en el programa de piscicultura agrícola de la sabaleta (Brycon henni). Medellín (Colombia): Corpourabá,

Builes J, Uran A. 1974. Estudio del ciclo sexual de la Sabaleta Brycon henni Eigenmann 1913, su comportamiento y fecundación artificial. Actualidades Biológicas, 3(7): 1-12. http://aprendeenlinea.udea. edu.co/revistas/index.php/actbio/article/view/330722/20786977

Bunn SE, Arthington AH. 2002. Basic principles and ecological consequences of altered flow regime for aquatic biodiversity. Environmental Management, 30(4): 492-507. DOI: 10.1007/ s00267-002-2737-0

Chaves-Ulloa R, Umaña-Villalobos G, Springer M. 2014. Downstream effects of hydropower production on aquatic macroinvertebrate assemblages in two rivers in Costa Rica. Revista Biología Tropical, 62 (2): 179-201. DOI:https://doi.org/10.15517/rbt. v62i0.15787

Draštík V, Kubečka J, Tušer M, Čech M, Frouzová J, Jarolím O, Prchalová M. 2008. The effect of hydropower on fish stocks: comparison between cascade and non-cascade reservoirs. Hydrobiología, 609: 25-36. DOI 10.1007/s10750-008-9393-1

Dudgeon D, Arthington AH, Gessner MO, Kawabata Z, Knowler DJ, Lévêque CR, Naiman J, Prieur-Richard A, Soto D, Stiassny MLJ, CA Sullivan. 2006. Freshwater biodiversity: importance, threats, status and conservation challenges. Biological Reviews of the Cambridge Philosophical Society, 81(2): 163-182. DOI:10.1017/ S1464793105006950

Empresas Públicas de Medellín E.S.P. 2009. Levantamiento de línea base para el desarrollo de una prueba piloto para el control químico de malezas acuáticas en el embalse Porce II. Rionegro (Colombia): Universidad Católica de Oriente. Informe Final. Contrato No 29990433817.

Eschmeyer WN, Fricke R, van der Laan R, editors. 2017. Catalog of fishes: genera, species, references. San Francisco (CA): California Academy of Sciences. [fecha de acceso octubre 30, 2017] http:// researcharchive.calacademy.org/research/ichthyology/catalog/ speciesbyfamily.asp

Fisher RA, Corbet AS, Williams CB. 1943. The Relation Between the Number of Species and the Number of Individuals in a Random Sample of an Animal Population. Journal of Animal Ecology, 12(1): 42-58. http://www.jstor.org/stable/1411

Granado-Lorencio C. 2002. Ecología de Peces. Sevilla (España): Universidad de Sevilla, Secretariado de Publicaciones.

Granado-Lorencio C, Hernández-Serna A, Carvajal JD, Jiménez-Segura LF, Gulfo A, Álvarez FE. 2012. Regionally nested patterns of fish assemblages in floodplain lakes of the Magdalena river (Colombia). Ecology and Evolution, 2(6): 1296-1303. https:// doi.org/10.1002/ece3.238 
Gomes LC, Miranda LE. 2001. Riverine Characteristics dictate composition of fish assemblages and limit fisheries in reservoirs of the upper Paraná river basin. Regulated Rivers: Research and Management, 17: 67-79. https://doi.org/10.1002/10991646(200101/02)17:1<67::AID-RRR615>3.0.CO;2-P

Gutiérrez-Bonilla FP, Álvarez-León R. 2011. Los Cíclidos (Pisces: Cichlidae) en Colombia: introducciones, trasplantes y repoblaciones No. 33, Luna Azul, 33: 154-177. http://www. redalyc.org/pdf/3217/321727235013.pdf

Hammer Ø, Harper DAT, Ryan PD. 2001 PAST: Paleontological statistics software package for education and data analysis. Paleontologia electronica, [fecha de acceso octubre 21, 2015]; 4(1): 9. https://palaeo-electronica.org/2001_1/past/past.pdf

Jackson DC, Marmulla G. 2001. The influence of dams on river fisheries. En: G. Marmulla, editor. Dams, fish and fisheries: opportunities, challenges and conflict resolution. Roma: Food and Agriculture Organization of the United Nations. Fisheries Technical Paper 419. Rome. p. 1-44.

Jiménez-Segura LF, Álvarez-León R, Gutiérrez-Bonilla F, Hernández S, Valderrama-Barco M, Villa-Navarro F. 2011a. La pesca y los recursos pesqueros en los embalses colombianos. En: CA Lasso, F Gutiérrez, M Morales-Betancourt, E Agudelo, H RamírezGil, RE Ajiaco-Martínez editores. II. Pesquerías continentales de Colombia: cuencas del Magdalena-Cauca, Sinú, Canalete, Atrato, Orinoco, Amazonas y Vertiente del Pacifico. Serie Editorial Recursos Hidrobiológicos y Pesqueros Continentales de Colombia. Bogotá (Colombia): Instituto de Investigación de los Recursos Biológicos Alexander von Humboldt. p. 233-281.

Jiménez-Segura LF, Granado-Lorencio C, Gulfo A, Carvajal JD, Hernández A, Álvarez F, Echeverry JP, Martínez A, Palacio J. 2011b. Uso tradicional de los recursos naturales pesqueros y conservación de la biodiversidad en regiones tropicales subdesarrolladas: hacia un modelo de Ecología de la Reconciliación. Informe final. Medellín (Colombia): Universidad de Antioquia, Universidad de Sevilla, Agencia Española de Cooperación Internacional para el Desarrollo, Cormagdalena.

Jiménez-Segura LF, Arango A, Ríos MI, García-Alzate C. 2013. Distribución altitudinal de la ictiofauna en un río trans-andino en el norte de Suramérica. En: Memorias del XII Congreso colombiano de ictiología y III Encuentro Suramericano de ictiólogos. Universidad Javeriana, ACICTIOS.

Jiménez-Segura LF, Maldonado-Ocampo J, Pérez C. 2014. Gradiente de recuperación longitudinal en la estructura de la ictiofauna en un río andino regulado. Biota Colombiana, 15(2): 61-80. DOI: https://doi.org/10.21068/bc.v15i2.321

Larrahondo M. 1993. Aprovechamiento acuícola de embalses en Colombia. En: JR Juárez, E. Varsi editores. Avances en el manejo y aprovechamiento acuícola de embalses en América Latina y el Caribe. México: FAO. http://www.fao.org/docrep/field/003/ ab488s/AB488S04.htm\#ch3

Loaiza CA, Jiménez-Segura LF. 2013. Áreas y momentos de reproducción de algunos Characiformes en el área de influencia de los embalses Porce II y Porce III: Algunos Aspectos de las
Estrategias de Vida [Trabajo de Pregrado]. [Medellín (Colombia)]: Instituto de Biología, Universidad de Antioquia.

Londoño JP, Jiménez-Segura LF. 2013. Dinámica reproductiva de algunas especies de Siluriformes y Perciformes presentes en el ensamblaje de la íctiofauna del río Porce en los embalses Porce II y Porce III [Trabajo de Pregrado]. [Medellín (Colombia)]: Instituto de Biología, Universidad de Antioquia.

Magallanes MH, Tabares MM. 1999. Estudio de los efectos del proyecto hidroeléctrico Porce II sobre la fauna íctica. Medellín (Colombia): Empresas Públicas de Medellín, gerencia de generación de energía, división Porce II, Departamento Gestión ambiental. Informe final.

Maldonado-Ocampo JA, Vari RP, JS Usma. 2008. Checklist of the freshwater fishes of Colombia. Biota colombiana, 9(2): 143237. http://revistas.humboldt.org.co/index.php/biota/article/ view/201/200

MargalefR. 1983. Limnología. Barcelona (España): Ediciones Omega, S.A. Montoya-López AF, Carrillo LM, Olivera-Angel M. 2006. Algunos aspectos biológicos y del manejo en cautiverio de la Sabaleta Brycon henni Eigenmann, 1913 (Pisces: Characidae). Revista Colombiana de Ciencias Pecuarias, 19(2): 180 - 186. http://www. redalyc.org/pdf/2950/295022982010.pdf

Murphy BR, Willis DW. 1996. Fisheries techniques. $2^{\text {nd }}$ ed. Bethesda (U.S.A.): American fisheries society.

Neiffer DL, Stamper MA. 2009. Fish sedation, anesthesia, analgesia, and euthanasia: considerations, methods, and types of drugs. ILAR Journal. 50 (4): 343-360. https://vpresearch.louisiana.edu/sites/ research/files/NeifferFishSedation.pdf

Nilsson C, Reidy CA, Dynesius M, Revenga C. 2005. Fragmentation and flow regulation of the world's large river systems. Science, 308(5720): 405-408. DOI:10.1126/science. 1107887

Ortiz JF. 2002. Evolución del Desarrollo Hidroeléctrico en el Departamento de Antioquia. Medellín (Colombia): Escuela de Ingenieros de Antioquia; [fecha de acceso octubre 25, 2015]. http://fluidos.eia.edu.co/hidraulica/articuloses/historia/hidroe_ antioquia/index.htm

Petrere Jr M. 1996. Fisheries in large tropical reservoirs in South America. Lake and Reservoirs: Research and Management, 2(1-2): 111-133. https://onlinelibrary.wiley.com/doi/ pdf/10.1111/j.1440-1770.1996.tb00054.x

Pompeu PS, Godinho HP. 2006. Effects of extended absence of flooding on the fish assemblages of three floodplain lagoons in the middle Sao Francisco River, Brazil. Neotropical Ichthyology, 4(4): 427-433. http://dx.doi.org/10.1590/S167962252006000400006

Silva SM. 2008. Evaluación de la Calidad del Agua del Futuro embalse Porce III por la influencia de la descarga del embalse Porce II modelo de simulación de calidad del agua del futuro embalse Porce III. Revista Ingenierias Universidad de Medellín, 7(13): 21-37. http://revistas.udem.edu.co/index.php/ingenierias/article/ view/193/180

Sokal RR. 1995. Biometry the principles and practice of statistics in biological research Robert R. Sokal and F. James Rohlf. 3rd ed. New York: New York W.H. Freeman. 
Stanford JA, Ward JV. 2001. Revisiting the serial discontinuity concept. Regulated Rivers: Research and Management 17(4-5): 303-310. https://doi.org/10.1002/rrr.659

Thornton KW. 1990. Sedimentary processes. En: KW Thornton, BL Kimmel, FE Payne editores. Reservoir limnology: ecological perspectives. New York (Estados Unidos): John Wiley and Sons. p. 43-70.

Trahl JH. 1973. Alimento y alimentación de la Sabaleta (Characidae: Brycon henni) en el sistema del río Porce y algunas observaciones sobre su dieta en cautividad. Medellín: Instituto Nacional de los Recursos Naturales y Renovables del Ambiente-Inderena.

Umaña VG. 2006. Ciclo anual de estratificación y circulación en el Embalse Arenal, Costa Rica. Revista de Biología Tropical, 54(1): 257-263. http://www.redalyc.org/articulo.oa?id=44921046016

Universidad De Antioquia, Empresas Públicas De Medellín E.S.P. 2013. Ecología de la comunidad íctica en el río Porce, en el área de influencia de los embalses Porce II y Porce III. Informe Final. Medellín (Colombia): Universidad de Antioquia, Empresas Públicas de Medellín. Convenio Nº 2011000558.
Uran CLA, Torrente PA. 2001. Monitoreo de fauna íctica en el embalse Porce II, sus quebradas afluentes y comparación con la línea base. Medellín (Colombia): Empresas Públicas de Medellín, Gerencia de Generación de Energía, Subgerencia Ambiental. Contrato $\mathrm{N}^{\circ}$ 030412212

Vannote RL, Minshall GM, Cummins KW, Sedell JR, Cushing CE. 1980. The river continuum concept. Canadian Journal of Fisheries and Aquatic Sciences, 37(1): 130-137. https://doi.org/10.1139/880-017

Ward JV, Stanford JA. 1983. The serial discontinuity concept of lotic ecosystems. En: TD Fontaine, SM Bartell editores. Dynamics of Lotic Ecosystems. Ann Arbor (MI): Ann Arbor Scientific Publishers. p. 29-42.

Ward JV, Stanford JA. 1995. The serial Discontinuity Concept: Extending the model to Floodplain rivers. Regulated Rivers: Research and Management, 10(2-4): 159-168. https://doi. org/10.1002/rrr.3450100211

Welcomme RL. 1985. River Fisheries. Roma (Italia): FAO Fishery Resources and Environment Division. FAO Fisheries Technical Paper 262

Whittaker RH. 1972. Evolution and measurement of species diversity. Taxon 21(2-3): 213-51. doi:10.2307/1218190 